\title{
Surveying the current landscape of clinical genetics residency training
}

\author{
Caleb P. Bupp, MD'1, Laurie A. Demmer, MD² and Robert A. Saul, MD $^{3}$
}

Purpose: Further knowledge about medical genetics residency training structure and function could help advance this educational process.

Methods: Medical genetics residency program directors were surveyed about their trainees' backgrounds and skills as well as the recruitment and matching process.

Results: Previous resident training was predominantly in pediatrics (49\%). Average ratings of residents' beginning clinical knowledge (scale of $1-10$, minimal to superior) were: dysmorphology -3.5 , inborn errors of metabolism -2.5 , prenatal genetics -2.6 , and cancer genetics -2.8 . On average, four months of research were required for categorical residency and fifteen months for combined residency. For the 2011 transition to ERAS/NRMP, 69\% of program directors were extremely or somewhat prepared; however, $21 \%$ felt unprepared. The number of trainees at most institutions remained unchanged. $36 \%$ of respondents reported that ERAS/NRMP has had no impact on recruitment of trainees, and $26 \%$ felt it has had a slightly positive impact. Continued utilization was recommended by $71 \%$ while $5 \%$ disagreed.

Conclusion: Genetics residents come from diverse training backgrounds. Their education can be directed toward specific areas of perceived initial weakness. ERAS/NRMP has not drastically increased entrance into the field. Further discussions are merited regarding enhancement of medical genetics residency recruitment and training.

Key Words: education; match; program director; residency; training

Genet Med advance online publication 18 September 2014
Medical education seeks to foster and enrich clinicians and other health-care professionals, expanding their knowledge base and, consequently, that of the entire medical field. Promoting and expanding medical genetics training is a priority for the American College of Medical Genetics and Genomics (ACMG), evidenced by its involvement in various aspects of education, its Careers in Medical Genetics programs, and many other ventures. Another group involved in genetics education is the Association of Professors of Human and Medical Genetics, which seeks to promote human and medical genetics education and is home to the Medical Genetics Residency Program Directors Special Interest Group. The American Board of Medical Genetics and Genomics (ABMGG) has also been recognized by the American Board of Medical Specialties as a primary specialty board. Medical genetics is an Accreditation Council for Graduate Medical Education (ACGME)-recognized specialty, and the ACGME has published competencies and requirements for training. Resident placement into medical genetics residencies transitioned to the usage of Electronic Residency Application Service (ERAS) and National Resident Matching Program (NRMP), sometimes termed "the match," in 2011. Some data are available regarding genetics trainees from ACGME, ACMG, ABMG, ERAS, and NRMP. This study aimed to gather information from a more direct source-medical genetics residency program directors. Details regarding their residents' previous training, knowledge and skill levels, and the functionality of the ERAS/NRMP system were queried. The survey was intended to gather information about the current state of medical genetics residency and to discover potential areas for improvement.

\section{MATERIALS AND METHODS}

A questionnaire composed of 19 multiple-choice, short answer, and rating scale questions was designed to gather the data of interest. The questions were grouped by topic into four major categories: general information about the survey respondent, questions about incoming genetics trainees, information about laboratory and research involvement, and questions about the ERAS/NRMP system. Each question included additional free-text space offered for commenting on that specific question, and the end of the survey offered the opportunity to add any additional overall comments. The survey was reviewed and granted approval for distribution by the Self Regional Healthcare Institutional Review Board. Surveys were distributed at the program directors meeting of the 2013 Association of Professors of Human and Medical Genetics Workshop at Kiawah Island, South Carolina. They were collected anonymously after completion. A preaddressed envelope was also provided for later return if a respondent wished. An identical copy of the survey was sent by mail to programs not present at the meeting and addressed to the ACGME-designated program director. Instructions to participate in the survey

${ }^{1}$ Spectrum Health, Grand Rapids, Michigan, USA; ${ }^{2}$ Department of Pediatrics, Carolinas Medical Center, Charlotte, North Carolina, USA; ${ }^{3}$ Children’s Hospital, Greenville Health System, Greenville, South Carolina, USA. Correspondence: Caleb P. Bupp (caleb.bupp@spectrumhealth.org) 
online were also included with the mailed paper copy, and it was requested that the program director return the paper copy or complete the survey online. In addition, the link to the online survey was also posted to the medical genetics program director online discussion group. A list of all combined genetics residency programs was cross-referenced to the list of categorical medical genetics residency programs to attempt to ensure that every institution was offered representation in this project. Surveys were collected through the various methods described and the data from them were combined for review. Questionnaires completed by non-US training program directors were not included because of differences in the structuring of training and their nonparticipation in ERAS/NRMP.

\section{RESULTS}

At the time of the survey's distribution, there were 49 ACGMEapproved categorical medical genetics residency programs. Forty-two surveys were collected anonymously for analysis. Of those 42, 38 were paper copies and 4 were submitted electronically. Three surveys were removed from the study. One survey was completed by a laboratory training director, one was completed by a former program director, and the other was completed by a director of a Canadian training program. These three surveys were collected in person at the Association of Professors of Human and Medical Genetics Program Directors Meeting. Of the qualifying respondents, 35 identified themselves as program directors and 4 identified themselves as assistant program directors. The average tenure in the position was 5.1 years, with the longest being 19.5 years and the shortest being only 2 months.

In characterizing the current status at their institution, respondents reported an average of 2.74 total medical genetics residents. This number includes both 2-year categorical and combined genetics residencies. The largest number of trainees

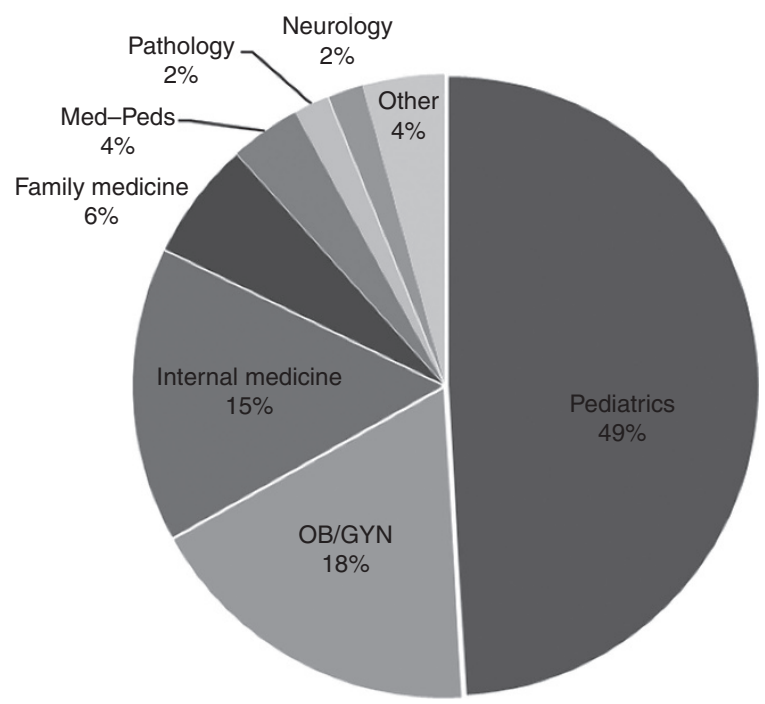

Figure 1 Previous training of categorical medical genetics residents who had been through training within the past 5 years $(n=163)$. The undefined $4 \%$ consisted of one trainee each from surgery, neurosurgery, radiology, radiation oncology, and ophthalmology residency. at one institution was 13 , whereas several institutions had none. When asked to describe the previous training of categorical trainees that had been through their training program within the past 5 years, the largest percentage (49\%) had trained in pediatrics. Obstetrics and gynecology accounted for $18 \%$, followed by internal medicine with $15 \%$, and family medicine with $6 \%$. Other previous training included internal medicine/ pediatrics (4\%), pathology (2\%), and neurology (2\%). A small number of other specialties made up the other $4 \%$, with one trainee each from surgery, neurosurgery, radiology, radiation oncology, and ophthalmology residency (Figure 1).

Respondents were asked to consider which training pathway best prepares a physician for medical genetics residency, and five options were given: (i) entering categorical medical genetics residency immediately after completing another residency; (ii) entering categorical medical genetics residency immediately after 2 years of any ACGME-accredited residency but not completing a previous residency; (iii) entering through combined genetics residency (i.e., pediatrics/genetics); (iv) having a period of medical practice between previous residency and entering medical genetics residency; and (v) no preference. The majority of respondents chose the first option-immediate entry after completing another residency $(65.8 \%)$. Entering by combined residency was selected by $10.5 \%$, and $3.2 \%$ had no preference.

The perceived knowledge and skill of incoming trainees was assessed. Program directors rated areas of genetic clinical knowledge on a scale from 1 to 10 (1, minimal; 10 , superior) for their trainees at the beginning of the residency. They were also asked to rate the ability of trainees to perform aspects of a genetic clinic visit using the same scale. The average scores given for trainees regarding their knowledge were as follows: dysmorphology, 3.5; inborn errors of metabolism, 2.5; prenatal genetics, 2.6; and cancer genetics, 2.8. Regarding ability, the average score for history taking was 6 and the average physical/ dysmorphology examination score was 4.2. Trainees' ability to create an assessment and plan averaged a score of 4 , and their genetic counseling capability score averaged 3.4 (Figure 2). Several respondents did note that, in their opinion, a resident's previous training had a significant impact on these ratings.

Program directors were asked to evaluate the experience and knowledge of their residents regarding laboratory genetics and genetic research. The mean reported time period for research required for categorical training programs was 4 months. Combined programs required more research (an average of 15 months). Respondents were also asked to rate their incoming trainees' levels of knowledge pertaining to three genetic laboratory areas on a scale from 1 to 10 (1, minimal knowledge; 10, superior knowledge). On average, trainees were given a score of 3 for molecular genetics, 2.7 for cytogenetics, and 2.2 for biochemical genetics (Figure 2). Sixty-five percent of respondents felt that an institution having laboratory fellowship training programs significantly enhanced clinical medical genetics residency. The rest of the program directors noted at least slight or moderate enhancement. 


\begin{tabular}{|c|c|c|c|c|c|}
\hline & & Average & Range & Median & Mode \\
\hline \multicolumn{6}{|l|}{$\begin{array}{l}\text { Clinical } \\
\text { knowledge }\end{array}$} \\
\hline & Dysmorphology & 3.5 & $1-6$ & 3.5 & 3 \\
\hline & Inborn errors of metabolism & 2.5 & $1-4$ & 2 & 2 \\
\hline & Prenatal genetics & 2.6 & $1-8$ & 3 & 3 \\
\hline & Cancer genetics & 2.8 & $1-6$ & 3 & 3 \\
\hline \multicolumn{6}{|l|}{ Clinical skill } \\
\hline & History taking & 6 & $1-8$ & 6 & 6 \\
\hline & Physical exam & 4.2 & $1-7$ & 4 & 3 \\
\hline & Assessment/plan & 4 & $1-7$ & 4 & 3 \\
\hline & Genetic counseling & 3.4 & $1-6$ & 3 & 3 \\
\hline \multicolumn{6}{|l|}{$\begin{array}{l}\text { Laboratory } \\
\text { knowledge }\end{array}$} \\
\hline & Molecular genetics & 3 & $1-8$ & 3 & 2 \\
\hline & Cytogenetics & 2.7 & $1-6$ & 3 & 3 \\
\hline & Biochemical genetics & 2.2 & $1-5$ & 2 & 2 \\
\hline
\end{tabular}

Figure 2 Program director ratings for various aspects of knowledge and skills of their incoming trainees. Program directors were asked to use a scale from 1 to 10 (1, minimal; 10 , superior).

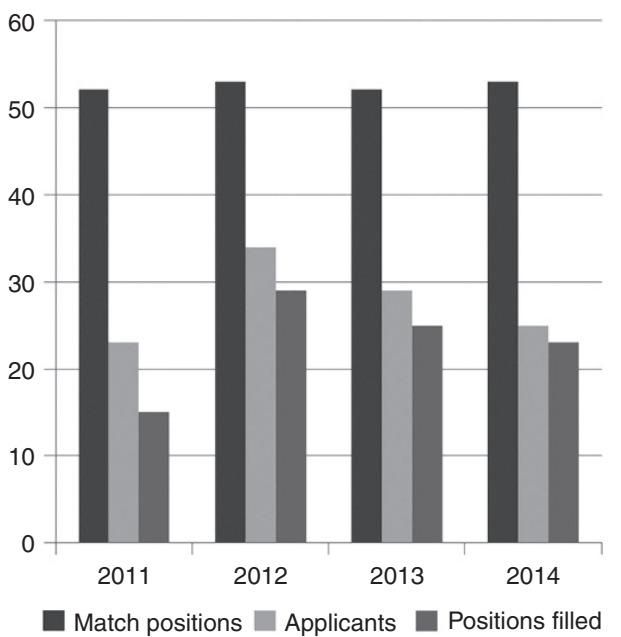

Figure 3 Data from National Resident Matching Program since initial utilization for categorical genetics residency in 2011. 52.5 categorical match positions have been available on average per year, with the average number of applicants being 27.75. An average of 29.5 positions remained unfilled, with a few applicants each year not matching at all.

Finally, the utilization and success of the ERAS/NRMP system were discussed. When asked how much this has impacted recruitment of physicians into the field of medical genetics, $19 \%$ felt it was a significant enhancement, $31 \%$ noted slight enhancement, $42 \%$ felt it had no impact, and $8 \%$ felt it had a negative impact. When ERAS/NRMP was initially implemented, $69 \%$ felt somewhat or extremely prepared to use the system, but $21 \%$ felt unprepared. Currently, $49 \%$ of respondents feel they are using the system comfortably and $49 \%$ experience only occasional difficulty. Since the start of ERAS/NRMP, 78\% of respondents have not seen a change in the number of trainees at their institution. A decrease was seen by $11 \%$ and an increase was seen by $5 \%$. Of those surveyed, $10 \%$ felt there was a decrease in applicant quality since the adoption of the ERAS/ NRMP, whereas $53 \%$ saw no difference. Thirty-two percent felt there has been some improvement. Continued usage of the ERAS/NRMP system was preferred by the majority of program directors (71\%); $5 \%$ felt it should be discontinued and $24 \%$ had no opinion regarding future usage. Open-response comments from the questionnaire regarding the ERAS/NRMP system spoke about hope that things would improve with time and that the system helped streamline the process while leveling the playing field between programs.

\section{DISCUSSION}

The ACGME has tracked the number of accredited medical genetics programs yearly. Over the past 10 years, nearly 50 programs each year have been accredited (an average of 48.6). ${ }^{1}$ Data are also gathered regarding the number of new program directors per year, ranging from 2 to 10 new directors per year, with an average of 5.5. ${ }^{2}$ Application of these figures implies that the average program will have a new program director nearly every 10 years. The average tenure of respondents for this study was only 5.1 years; however, assistant directors were also included in this project's collection. Based on this information, a program could proactively put mechanisms in place to adequately mentor and train assistant directors, anticipating eventual change in leadership.

According to ACGME data, since 2001, there have been an average of 79 total on-duty categorical medical genetics residents (post-graduate year (PGY) 1 and PGY2) per year. ${ }^{1}$ There have also been a mean of $\sim 23$ on-duty combined pediatrics/ genetics residents and five combined internal medicine/genetics residents (internal medicine/genetics data available beginning in 2003). The ABMGG currently notes 120 total on-duty residents: 30 PGY-1 and 37 PGY-2 categorical residents, 5 categorical residents beyond their second year, 34 pediatrics/ genetics residents, 2 internal medicine/genetics residents, and 12 maternal-fetal medicine/genetics residents (personal communication from the ABMGG). 
Data from the NRMP show that since the system was implemented in 2011, between 33 and 38 categorical medical genetics programs have participated each year. On average, only 12.5 programs completely filled their match positions. There have been a total of 52.5 available match positions on average, yet the average number of applicants is significantly lower at 27.75 each year. $^{3}$ Despite an initial increase in categorical applicants from the first year to the second year of ERAS/ NRMP, the number of applicants has since leveled off. An average of 29.5 positions remained unfilled, with a few applicants each year not matching at all (Figure 3). Over the 4 years of NRMP/ERAS utilization, there has been little overall change in the number of categorical trainees, which is consistent with the responses gathered in this survey.

There have been, on average, 11.75 combined pediatrics/ genetics and 2.25 combined medicine/genetics programs participating in NRMP since 2011. Each year, a mean of 2.75 pediatrics/genetics programs and 1 medicine/genetics program did not fill to capacity. An average of 8.5 and 1.75 yearly match positions were available, with the mean number of applicants being 17.75 for pediatrics/genetics and 6.5 for medicine/genetics. ${ }^{4}$

Some residents may enter training outside of ERAS/NRMP. ABMGG data show that 30 categorical PGY-1 residents began training in 2013. According to the NRMP, 25 residents matched to programs that year. This means that $83 \%$ of positions were filled through ERAS/NRMP and 17\% were not (personal communication from the ABMGG).

Written competencies for physician medical geneticists have been created by the ACMG, including overarching and discipline-specific competencies. ${ }^{5}$ These classifications were somewhat different than the classifications used in this survey, but they reflect a similar viewpoint. One specific area that was not explicitly commented on in the competencies was laboratory genetics education. Hopefully, the results of this survey will serve as a companion to the ACMG competencies by showing where trainees begin in their residencies. The comparatively lower rated areas of knowledge and skill could be viewed as areas in need of additional emphasis and review for learners. For example, the lowest rated area of clinical knowledge was for inborn errors of metabolism and the lowest rated area of laboratory knowledge was for biochemical genetics; this may suggest that these areas may need additional emphasis or focus for trainees.

The ABMGG administers an annual in-training examination to trainees during the 8 th month of the residency year. On the 2013 and 2014 examinations, the biochemical genetics content area was not the lowest scored area for clinical categorical firstyear and second-year residents. In 2013, the lowest scored area for first-year categorical residents was prenatal genetics, and in 2014 it was clinical genetics. For second-year categorical residents, the lowest scores came in basic principles in 2013, and in clinical genetics in 2014 (personal communication from the ABMGG). Somewhat large standard deviations for scoring and testing being performed after a period of 8 months of training make these data difficult to directly compare with those of this study. As a final added note for this study, comparative ratings from program directors based on their residents' knowledge at the end of training would be useful. Also, self-reported ratings from residents in these same areas could provide additional information that individual programs might find helpful in refining their curriculum.

The insights gained from directors' implementation and usage of ERAS/NRMP could also prove useful. During the first year of use, the fewest number of programs filled, there were the most unfilled positions, and the fewest number of applicants were matched. In the 3 years since that initial implementation, these numbers have improved overall somewhat from the first year but have since remained relatively stable. Four years of match data suggest that utilizing ERAS/NRMP may not improve the recognized sluggish growth of ABMG certifications. One of the original intents of medical genetics' participation in ERAS/NRMP was to provide a level playing field for all programs and applicants. This sentiment was reflected by several survey respondents. This program director survey shows that despite some initial utilization problems, directors are using ERAS/NRMP with minimal problems. Thus, utilization appears to be nearing optimization. With $71 \%$ believing that it should be continued and $24 \%$ with no opinion, and the overall lukewarm response to ERAS/NRMP seen in this survey, it appears that program directors may recognize the limitations of ERAS/NRMP to improve the recruitment of geneticists.

The data showing more categorical genetics residents previously trained in pediatrics likely make anecdotal sense to most practitioners. However, a more detailed breakdown of the previous training pathways may help broaden recruitment strategies to include a larger variety of specialties. Certainly, the vast applications that genetics offers to the practice of medicine would allow a physician of any training background the opportunity for meaningful study in his or her particular area of expertise. Most respondents believed that completing a previous residency in another specialty before entering a dedicated genetics residency was the preferred pathway to a career in genetics; this is a finding that merits specific mention. Combined pediatrics/genetics residencies have recently been changed to consist of 4 years of training, a decrease from the previous requirement of 5 years. Combined internal medicine/ genetics residencies may also follow suit in the future. However, only $10.5 \%$ of program directors felt combined residency was the best training pathway. In addition, a 2014 change in program requirements stated that only 1 year of previous ACGME training will be required before entering genetics residency. ${ }^{6}$ Going forward, it will be important to carefully balance the need for additional trainees in genetics with ensuring that the training is adequate and thorough.

There is no formalized time frame dictated by the ACGME for the research component of categorical medical genetics residency. There is a requirement for scholarly activity, including submission of an abstract, presentation, or article for publication. ${ }^{7}$ Each training program is able to create its own guidelines and regulations for research, which can be tailored to the 
program's structure. Some requirements exist for combined genetics residencies. Maternal-fetal medicine/genetics combined residencies require 12 months of dedicated research ${ }^{8}$ (a decrease from 18 months previously required), ${ }^{9}$ pediatrics/genetics has a recommendation for 6 months (during a 4 -year combined residency time period), ${ }^{10}$ and internal medicine/genetics does not have stated guidelines. ${ }^{11}$ Differences in required research time vary based on the type of training program, and hopefully the sharing of average time periods in this study can serve to provide guidance and comparison for individual programs tasked with this determination.

The willingness and graciousness with which program directors past and present facilitated and participated in this project serve to brighten the outlook for medical genetics training. The large percentage of programs that participated speaks to their engagement and vested interest in trainees and their education. The ACMG has also shown its willingness to engage in the topic of improving education. The findings presented here can help to further the discussion about how best to recruit and train genetics residents, as well as how to expand and enrich this cycle in the future.

\section{ACKNOWLEDGMENTS}

The authors thank the Association of Professors of Human and Medical Genetics Medical Genetics Residency Program Directors Meeting and the Medical Genetics Residency Program Directors Listserv for facilitating participation in this project. Additional thanks go to V. Reid Sutton and Gerald Feldman for their support, and to Roger Stevenson, Steven Skinner, Neena Champaigne, Sara Sarasua, and Fran Annese, for their suggestions and contributions.

\section{DISCLOSURE}

The authors declare no conflict of interest.

\section{REFERENCES}

1. Accreditation Council for Graduate Medical Education. Number of Accredited Programs for the Current Academic Year (2013-2014) United States. http:// www.acgme.org/ads/Public/Reports/ReportRun?Reportld=3\&CurrentYear $=20$ 13\&AcademicYearld=2013. Accessed 9 April 2014.

2. Accreditation Council for Graduate Medical Education. Number and Percent of New Program Directors by academic year (excluding combined programs) (7/1/2013-6/30/2014). http://www.acgme.org/ads/Public/Reports/ReportRu $n$ ?Reportld=6\&CurrentYear=2013\&Academic Yearld=2013. Accessed 9 April 2014

3. National Resident Matching Program. Results and Data-Specialities Matching Service, 2014 Appointment Year. http://www.nrmp.org/wp-content/ uploads/2013/08/National-Resident-Matching-Program-NRMP-Results-andData-SMS-2014-Final.pdf. Accessed 9 April 2014

4. National Resident Matching Program. Results and Data-2014 Main Residency Match. http://www.nrmp.org/wp-content/uploads/2014/04/Main-MatchResults-and-Data-2014.pdf. Accessed 11 June 2014.

5. Korf BR, Irons M, Watson MS. Competencies for the physician medical geneticist in the 21st century. Genet Med 2011;13:911-912.

6. Accreditation Council for Graduate Medical Education. ACGME Program Requirements for Graduate Medical Education in Medical Genetics. http:// www.acgme.org/acgmeweb/Portals/0/PFAssets/ProgramRequirements/130_ medical_genetics_07012014.pdf. Accessed 16 April 2014.

7. Accreditation Council for Graduate Medical Education Review Committee for Medical Genetics. Frequently Asked Questions: Medical Genetics. http:// www.acgme.org/acgmeweb/Portals/0/PDFs/FAQ/130_medical_genetics_ FAQs_07012014.pdf. Accessed 9 April 2014.

8. Board of Medical Genetics and Genomics. Program Requirements for Combined Maternal-Fetal Medicine Fellowship/Medical Genetics Residency. http://www. abmg.org/pdf/Program\%20Req\%20Combined\%20MFM-MG_05222014. pdf. Accessed 16 June 2014

9. Society for Maternal-Fetal Medicine. Directory of Fellowship Programs in Maternal-Fetal Medicine and MFM-Genetics. https://old.smfm.org/ attachedfiles/Directory2009.pdf. Accessed 18 June 2014.

10. American Board of Medical Genetics and Genomics. Guidelines for Combined Training in Pedatrics and Medical Genetics Leading to Dual Certification. http://www.abmg.org/pdf/Medgenetics\%202013\%20FINAL.pdf. Accessed 16 June 2014.

11. American Board of Medical Genetics and Genomics. Guidelines for Combined Internal Medicine/Medical Genetics Residency Training. http://www.abmgg. org/pdf/5-yr\%20IM-MG\%20Program\%20Requirements_06302014.pdf. Accessed 16 June 2014. 